\title{
Impact of smoking on the quantity and quality of antibodies induced by human papillomavirus type 16 and 18 AS04-adjuvanted virus-like-particle vaccine - a pilot study
}

Proscovia B Namujju ${ }^{1,2^{*}}$, Emma Pajunen ${ }^{1}$, Aline Simen-Kapeu², Lea Hedman, ${ }^{3,4}$ Marko Merikukka², Helja-Marja Surcel ${ }^{2}$, Reinhard Kirnbauer ${ }^{5}$, Dan Apter ${ }^{6}$, Jorma Paavonen ${ }^{7}$, Klaus Hedman $^{3,4}$ and Matti Lehtinen ${ }^{1}$

\begin{abstract}
Background: The AS04-adjuvanted bivalent L1 virus-like-particle (VLP) vaccine (Cervarix ${ }^{\mathrm{TM}}$ ) against infection with human papillomavirus (HPV) types 16/18 holds great promise to prevent HPV16/18 infections and associated neoplasias, but it is important to rule out significant co-factors of the neoplasias like smoking.

Methods: We conducted a pilot study to compare the quantity and quality of HPV16/18 antibody response at baseline and 7 months post vaccination in 104 non-smoking and 112 smoking female participants vaccinated at 0 , 1 and 6 months with Cervarix ${ }^{\mathrm{TM}}$ (55 and 48 study participants) or with Hepatitis A vaccine (HAVRIX ${ }^{\mathrm{TM}}$ ) (48 and 64 participants, respectively). These 216 women were a sub-sample of 4808 baseline 16- to 17-year old Finnish women initially enrolled in the double-blind, randomized controlled phase III PATRICIA trial. Following end-of-study unblinding in 2009 they were randomly chosen out of all the participants of the three major Finnish PATRICIA study sites in the Helsinki metropolitan area (University of Helsinki, $\mathrm{N}=535$, and Family Federation Finland, $\mathrm{N}=432$ ) and Tampere (University of Tampere, $N=428$ ). Following enrolment, serum samples were collected at month 0 and month 7 post 1st vaccination shot, and were analysed for levels and avidity of IgG antibodies to HPV16 and HPV18 using standard and modified (4 M urea elution) VLP ELISAs.

Results: We found that at month 7 post vaccination women who smoked (cotinine level $>20 \mathrm{ng} / \mathrm{ml}$ ) had levels of anti-HPV16/18 antibodies comparable to those of non-smoking women. Low-avidity HPV16/18 lgG antibodies were observed in $16 \%$ of the vaccinated women, and active smoking conferred a three-fold increased risk (95\% Cl 1.0-9.3) of having the low-avidity antibodies.
\end{abstract}

Conclusion: Our data suggest that while smoking does not interfere with the quantity of vaccine-induced peak $\lg G$ levels, it may affect the avidity of IgG induced by HPV16/18 vaccination.

Keywords: Antibody, Avidity, Human papillomavirus, AS04 adjuvanted vaccines, Cotinine, PATRICIA, Finland

\footnotetext{
* Correspondence: bazanyanengo@yahoo.co.uk

${ }^{1}$ School of Health Sciences, University of Tampere, Tampere, Finland

${ }^{2}$ National Institute for Health \& Welfare, P.O. Box 310, 90101 Oulu, Finland

Full list of author information is available at the end of the article
} 


\section{Background}

Infection with high-risk (hr) types of human papillomavirus (HPV) is the major cause of cervical cancer (CC) [1]. The necessary role of hrHPV infections in CC and other HPV related cancers provides an opportunity to significantly reduce associated disease burden by prophylactic vaccines [2] with proper coverage/herd immunity $[3,4]$.

Important determinants of vaccine efficacy are the quantity and quality of the B-cell response. The AS04adjuvanted HPV16/18 L1 virus-like-particle (VLP) vaccine induces high titer antibodies in adolescent and young women and men [5,6], able to neutralize the virus $[7,8]$, and detectable up to 8.4 years post vaccination [9]. The immune responses are, however, not homogenous, eg. a proportion of HPV-16/18 vaccinated women, those with significantly lower serum antibody levels, had no detectable cervical antibodies 4 years post vaccination [6]. Furthermore, not all vaccinees develop high avidity antibodies, and the avidities and levels of the neutralizing antibodies correlate only moderately [10]. High avidity of HPV vaccine induced antibodies may indicate successful priming for long-term memory responses as previously suggested by Scherpenisse [11].

Smoking women have an impaired humoral immune response to HPV16/18 infections [12]. Smoking has also been associated with decreased clearance of persistent HPV lesions [13]. Furthermore, epidemiological studies have indicated that tobacco smoking is an independent risk factor for CC [14]. The effect of smoking on vaccine efficacy and effectiveness has been studied in influenza vaccine trials [15], but its influence on the HPV vaccine response is unknown. In this pilot study, we compared the quantity and quality of HPV16/18 antibody responses at baseline and seven months post vaccination in smokers and nonsmokers vaccinated with three doses of AS04-adjuvanted HPV16/18 VLP vaccine or Hepatitis A vaccine.

\section{Methods \\ Study participants}

Enrolment for the PApilloma TRIal against Cancer In young Adults (PATRICIA (study trial number -580299/ 008)) study took place from April 2004 to May 2005 in Finland [16]. Healthy women aged 16-17 years were eligible to participate in the Finnish arm of this study with no exclusion criteria with regard to lifetime number of sexual partners before study enrolment [16,17]. Individuals with intact cervix, and agreeing to adequate contraception (barrier methods in combination with a spermicide, or hormonal contraception) over the vaccination period were eligible for inclusion. Exclusion criteria were limited to a history of colposcopy, pregnancy or breastfeeding, as well as autoimmune diseases and immunodeficiency. Informed consent was obtained from each participant at study baseline including later linkage to the Finnish Maternity Cohort (FMC) for the identification of serial serum samples post vaccination. The study protocols, recruitment material and informed consent forms were approved by the Finnish National and Pohjoispohjanmaan Sairaanhoitopiirin ethical review committees, and the retrieval of serum samples from the FMC repository by the National Institute for Health \& Welfare.

\section{Study design}

The PATRICIA study was a phase III double-blind, randomized controlled trial. In Finland, a total of 4,808 participants, were randomized in a 1:1 fashion with an internetbased centralized randomisation system, received either the AS04-adjuvanted HPV16/18 vaccine ((GlaxoSmithKline Biologicals, Rixensart, Belgium), (Each dose of HPV-16/18 L1 VLP AS04-adjuvanted candidate vaccine (Cervarix ${ }^{\mathrm{Tm}}$ ) contained $20 \mathrm{mg}$ each of HPV16 and HPV18 L1 proteins self-assembled as VLPs and adjuvanted with AS04 (50 $\mu \mathrm{g}$ 3-O-desacyl-40-monophosphoryl lipid A [MPL] and $500 \mu \mathrm{g}$ aluminium hydroxide)) or, a control hepatitis A vaccine ((GSK Biologicals), Each dose of the control hepatitis A vaccine contained 720 ELISA units (EU) of inactivated hepatitis $\mathrm{A}$ antigen and $0.5 \mathrm{mg}$ aluminium hydroxide)) to provide a health benefit for all participants and ensure double-blinding. Allocation of treatment numbers was stratified by study site and age.

A random sample of 216 study participants with unblinded individual vaccine allocation were selected for this study from the three largest Finnish study sites: Finnish Family Federation, Helsinki (432 participants, PI DA), University of Helsinki, Helsinki (535 participants. PI JP) and University of Tampere, Tampere (428 participants, PI ML). Serial serum samples accounting for the FMC-based long-term follow-up were available for 51, 97 and 103 participants, respectively, altogether for 251 of the total 1395 participants. The vaccines were supplied double-blinded in identical $0.5 \mathrm{ml}$ pre-filled syringes and administered into the deltoid muscle on a 0,1 , and 6-month schedule.

\section{Serologic evaluation}

Blood samples from the study participants were collected at baseline and at months 7 post vaccination, and an aliquot of each sample was stored at the National Institute for Health and Welfare laboratory in Oulu, Finland. Baseline and month 7 post vaccination trial serum samples were evaluated for HPV16 and HPV18 antibodies using a typespecific enzyme-linked immunosorbent assay (ELISA) as described [18-20]. Dilutions $1 / 30,1 / 300$ and $1 / 3000$ were used to identify the linear part of the absorbance reactions for expression of the results as OD values.

\section{Avidity measurement}

The avidity of IgG antibodies to HPV16 and HPV18 was evaluated in the HPV vaccine group $(n=103)$ using modified VLP ELISAs [21] Namujju et al. unpublished, 
(The VLPs were kindly donated by Dr Francis Dessy, GSK biologicals, Rixensart, Belgium). First, we screened the HPV16/18 vaccine induced antibodies for low avidity by single-dilution methods, using in parallel $4 \mathrm{M}$ urea or $1 \mathrm{M}$ ammonium thiocyanate as chaotropic agents. The month 7 post vaccination samples were diluted 1:1000 in assay diluent (PBS $+10 \%$ fetal bovine serum). Each sample was added in duplicate to a plate, one well in the plate was washed with PBS $+0.05 \%$ Tween 20 (PBST), and another well in the plate with either of the chaotropic agents. Blank well (assay diluent), negative and positive control pools for HPV6/11/16/18/31/33/45 natural infection derived antibodies, and acute-immunity (obtained from seroconverters within 6 months of natural infection) pools for HPV16 or HPV18 antibodies diluted 1:30 were included in each plate. After 2-hour incubation at room temperature (RT) the plates were washed three times with PBST and then (200 ul of) PBST was added in half of the plate, and (200 ul of) $4 \mathrm{M}$ urea or $1 \mathrm{M}$ ammonium thiocyanate in the other half for $15 \mathrm{mi}$ nutes. The plates were again washed two times with PBST, after which the wells treated with the chaotropic reagents were rinsed extra two times with PBST. Thereafter the standard VLP ELISA procedures was followed [21]. The avidity index (AI) was calculated using the formula optical density (OD) of the chaotrope-treated well/OD of the PBST-treated well) $\times$ 100 . The cut-off for low avidity (40\%) was the mean +3 standard deviations of acute- immunity controls.

Samples with results of low avidity in the singledilution screening test were further analyzed by a serum titration approach [21-23], Namujju et al. unpublished, albeit using $4 \mathrm{M}$ urea rather than $6 \mathrm{M}$ urea. The samples were serially diluted at four fold steps; A) $1: 100$, B) $1: 400$, C) $1: 1600$, D) $1: 6400$ and E) $1: 25600$. After 2-hour incubation at RT, one half of the plate was washed with $4 \mathrm{M}$ urea, and another half with PBST. Thus, from each sample two IgG-end-point titres at a cut-off of 0.200 (urea +/ urea -) were obtained. Their ration - representing IgG avidity - was calculated with curve-fitting software [24].

\section{Cotinine measurements}

Serum samples were measured for cotinine using an immunoassay method (OraSure Technologies, Bethlehem, PA, USA) carried out as a quantitative assay based on the competition between free cotinine in the sample and horseradish peroxidase-labeled cotinine. Cotinine was quantified spectrophotometrically at $450 \mathrm{~nm}$ and $630 \mathrm{~nm}$ relative to a standard curve. The assay's sensitivity is $95 \%-$ $97 \%$ and specificity $99 \%-100 \%$ [25,26]. A cotinine level of $20 \mathrm{ng} / \mathrm{ml}$ was used as an indicator of an active smoker, according to the manufacturer.

\section{Statistical analyses}

The pilot immunoanalyses included women who met eligibility criteria, received all vaccination shots and complied with the protocol procedures. Seropositivity rates of HPV16 and HPV18 were calculated. The Mann-Whitney test was used to compare the mean absorbances of antiHPV16 and HPV18 antibodies between non-smokers and smokers in vaccine and control groups. For each antigen the mean absorbance level and standard deviation $( \pm \mathrm{SD})$ were reported. Kappa-coefficients were calculated to evaluate consistency of the two parallel screening methods for the detection of low avidity HPV16/18 antibodies. The proportions of low-avidity HPV16 and HPV18 antibodies by the titration method were calculated, and the crude odds ratios (OR) with 95\% confidence interval (CI) of lowavidity antibodies according to smoking status were estimated using logistic regression. All statistical analyses were performed with SPSS 16.0 and STATA 8 (Stata Corp., College, Texas, USA).

\section{Results}

Demographic characteristics of the entire vaccinated cohort have been published previously [17]. In the present pilot study, we included 216 Finnish trial participants with unblinded vaccine codes, and who had complied with the full three-dose vaccination schedule. Out of 103 participants in the HPV16/18 vaccine group, $46.6 \%$ (48) were smokers compared to $56.6 \%$ (64) in the control group $(\mathrm{n}=113)$.

Mean anti-HPV16 and HPV18 antibody levels at month 7 post-vaccination were very high in the HPV16/18 vaccine group as compared to the control (HAV vaccine) group or to baseline (Table 1). When the anti-HPV16 and anti-HPV18 antibody levels between non-smokers and smokers were compared, the mean absorbance of antiHPV16 antibodies was $1.97( \pm 0.78)$ among non-smokers and $1.88( \pm 0.73)$ among smokers. Mean absorbance of anti-HPV18 antibodies was $1.44( \pm 0.85)$ among nonsmokers and $1.36( \pm 0.76)$ among smokers. The observed differences between the smokers and non-smokers were not statistically significant (Table 1).

We also evaluated in the HPV16/18 vaccine group the quality, i.e. the avidity of vaccine-induced HPV16 and HPV18 antibodies. In the screening phase, where $4 \mathrm{M}$ urea or $1 \mathrm{M} \mathrm{NH4CSN} \mathrm{were} \mathrm{used} \mathrm{as} \mathrm{chaotropic} \mathrm{agents,}$ reasonably high kappa-coefficients of consistency for presence of low avidity HPV antibodies ( 0.7 for low avidity HPV16 antibodies; 0.6 for low avidity HPV18 antibodies) were observed in 27 and 17 individuals, respectively.

By the reference approach (titration method using $4 \mathrm{M}$ urea), 13 out of the 27 samples with screen-detected HPV16 samples were confirmed to have low avidity, whereas three of the 17 screen-detected HPV18 samples had low-avidity HPV18 antibodies (Table 2). Eleven of 
Table 1 Comparison of mean absorbance level and mean antibody titers with standard deviation (SD) of anti-HPV16 and HPV18 antibodies between non-smokers and smokers by vaccination group

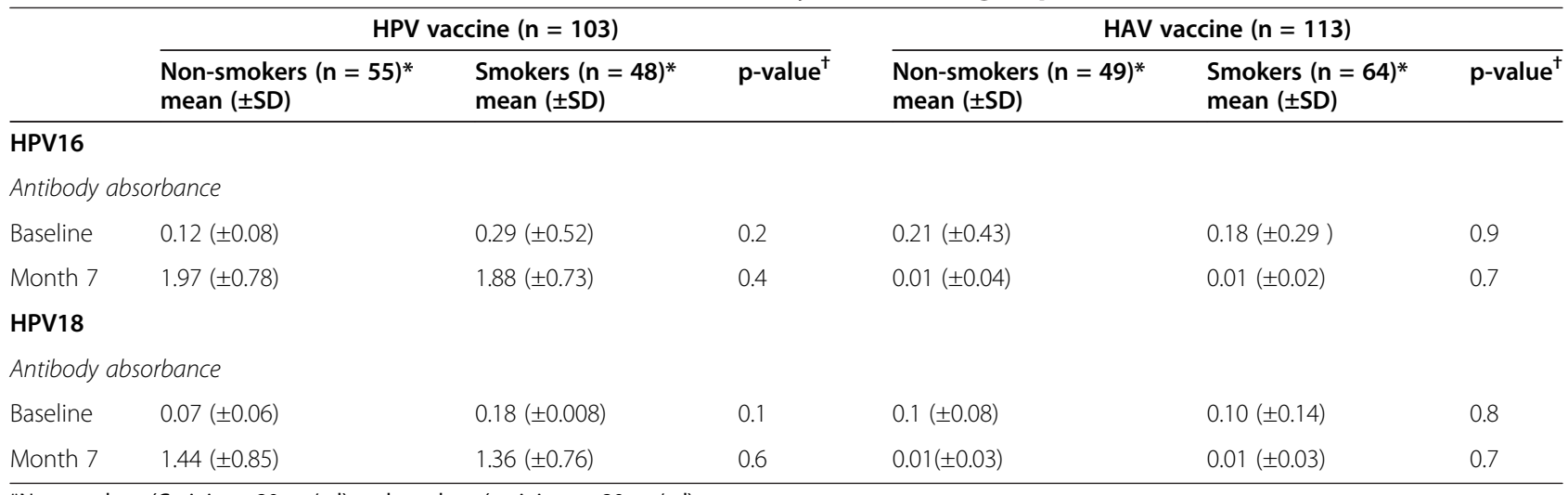

*Non-smokers (Cotinine $<20 \mathrm{ng} / \mathrm{ml}$ ) and smokers (cotinine $>=20 \mathrm{ng} / \mathrm{ml}$ ).

${ }^{\dagger} \mathrm{p}$-value derived from Mann-Whitney test to evaluate mean absorbance difference between non-smokers and smokers.

the 16 individuals with low-avidity HPV16/18 antibodies were smokers. They tended to have an increased risk of having low avidity HPV16/18 antibodies (OR 3.0, 95\% confidence interval (1.0-9.3) (Table 2).

\section{Discussion}

Evaluating the influence of HPV co-factors, particularly tobacco smoking, in the induction of robust post-vaccination anti-HPV immune response is timely [2]. We found that vaccinated young women, who smoked had similar levels of anti-HPV16 and HPV18 antibodies compared to vaccinated, non-smoking women 7 months post-vaccination, whereas the avidity of HPV16/18 antibodies might be inferior in smokers.

In contrast to natural HPV infection, a prophylactic cervical cancer vaccine should induce long-term immune response with high and sustained local and systemic antibody levels [27]. The adjuvanted HPV16/18 induces significantly increased humoral response to the vaccine antigens [28]. In addition to $100 \%$ seroconversion rates, extremely high antibody levels following Cervarix ${ }^{\mathrm{TM}}$ vaccination have been evident since the interim analysis of the
PATRICIA study, a large phase III trial of the AS04adjuvanted bivalent HPV16/18 vaccine $[16,17,29]$. The geometric titers of vaccine induced antibodies against both HPV16 or HPV18 antigens are substantially higher and longer lasting than those in natural HPV16/18 infections at all time points post vaccination/infection $[9,30]$.

Our pilot study in young women demonstrated no significant quantitative differences in vaccine-induced humoral immune response to HPV16/18 L1 VLPs among smokers compared to nonsmokers. The proportion of smoking women with apparently low avidity HPV16 antibodies was, however, approximately two times higher than in non-smoking women. Previous studies on HPV16/18 vaccine induced (maturation of) antibody avidity disagree on the existence of lowavidity antibodies [10,11,31]. In those studies confounding factors, e.g., differences in laboratory methods or smoking have not been considered. The applied chaotropic agent concentrations, re-optimized in our lab, had a minimal effect on the performance of the HPV16/18 VLP antigens in the standard ELISA assay (Namujju et al. unpublished).

Table 2 Detection of persistent low avidity HPV16 or HPV18 antibodies at month 7 stratified by smoking status

\begin{tabular}{llll}
\hline & Low avidity $(=<\mathbf{4 0 \%})$ & High avidity $(>\mathbf{4 0} \%)$ & OR, $\mathbf{9 5 \%}$ Cl \\
\hline HPV16 & & & \\
Non-smokers $(n=55)$ & $4(31 \%)$ & $51(57 \%)$ & $39(43 \%)$ \\
Smokers $(n=48)$ & $9(69 \%)$ & & $2.9(0.8-10.3)$ \\
HPV18 & & $54(54 \%)$ & 1 \\
Non-smokers $(n=55)$ & $1(33 \%)$ & $46(46 \%)$ & $2.8(0.2-31.6)$ \\
Smokers $(n=48)$ & $2(67 \%)$ & & 1 \\
HPV16/18 & & $50(57 \%)$ & $3.0\left(1.0^{*}-9.3\right)$ \\
Non-smokers $(n=55)$ & $5(31 \%)$ & $37(43 \%)$ & \\
Smokers $(n=48)$ & $11(69 \%)$ & & \\
\hline
\end{tabular}

*Exact value $=0.95$, and $40 \%$ is the cut-off of low avidity. 
Tobacco smoking, assessed via serum cotinine quantification, has been shown to have an adverse effect on immune response to natural HPV16/18 infections among young women followed-up for more than 5 years [12]. Although the underlying mechanism is unclear, researchers have demonstrated reduction in cervical density of Langerhans cells among smokers [32,33]. Tobacco smoking has also been associated with an increased probability of acquisition and a decreased probability of clearance of HPV infection $[34,35]$ as well as decreased clearance of cervical lesions [13].

Cotinine is a highly sensitive and specific marker of current active or passive exposure to tobacco smoke [36-38]. The use of serum cotinine rather than questionnaire data integrates a number of aspects of the exposure including tobacco composition, uptake, distribution, and individual differences in metabolism [38]. Furthermore, it is the inhaled dose of tobacco smoke that is directly related to the development of tobacco-related disorders [26]. Thus, biochemical assessment of tobacco exposure underlines the validity of our observations.

Duration of HPV vaccine induced immune response and its sustained efficacy in preventing HPV infection and cervical neoplasia are under close surveillance [2]. Some studies have shown that vaccine induced antibody levels decline with time. Although this has been suggested also for the bivalent HPV16/18 vaccine we have not observed it in our current study due to the short duration. In a study by Olsson et al. [39] it was suggested that although the HPV18 antibodies declined over time, the vaccinees remained protected, pointing to the importance of factors other than antibody levels in longterm vaccine protection. Sustainable, high avidity IgG response could be a surrogate marker or indirect indicator of T-cell help post-vaccination, which may or may not be affected by smoking habit. Our study was limited by the small numbers of study participants.

\section{Conclusion}

Our pilot results suggest that smoking may not interfere with the quantity, but affects the quality of HPV16/18 vaccine-induced peak IgG antibodies. Our findings warrant further large studies on the quality and duration of HPV16/18 vaccine induced antibody response.

\section{Competing interests}

$\mathrm{ML}$ and JP have obtained grants from Merck \& Co. Inc., and GlaxoSmithKline Biologicals through their employers. The other authors have no conflicts of interest to disclose.

\section{Authors' contributions}

PBN, ASK, HMS, ML designed the study; PBN, EP, LH conducted antibody avidity analysis; PBN, MM, ASK, ML participated in statistical analysis; PBN, EP, ML drafted manuscript, PBN, EP, ASK, HL, MM, HMS, RK, DA, JP, KH, ML contributed scientifically to the manuscript and approved the final manuscript.

\section{Acknowledgements}

We are grateful to Eveliina Jalonen and Saeed Shafti-Keramat for excellent technical help

\section{Funding}

This research was supported by grants from Finnish Cancer Society and to RK from the Vienna Science and Technology Fund (WWTF) (Life Science Call 2011) (LS11-006)

\section{Author details}

${ }^{1}$ School of Health Sciences, University of Tampere, Tampere, Finland. ${ }^{2}$ National Institute for Health \& Welfare, P.O. Box 310, 90101 Oulu, Finland.

${ }^{3}$ Haartman Institute, University of Helsinki, Helsinki, Finland. ${ }^{4}$ Helsinki University Central Hospital Laboratory Division, Helsinki, Finland. ${ }^{5}$ Department of Dermatology (DIAID), Medical University Vienna, Vienna, Austria. ${ }^{6}$ Family Federation Finland, Helsinki, Finland. ${ }^{7}$ University of Helsinki, Helsinki, Finland.

Received: 18 December 2013 Accepted: 30 June 2014

Published: 11 July 2014

\section{References}

1. IARC working group on the evaluation of carcinogenic risks to humans: Human papillomaviruses. IARC Monogr Eval Carcinog Risks Hum 2007, 90:1-636.

2. Lehtinen M, Dillner J: Clinical trials of human papillomavirus vaccines and beyond. Nat Rev Clin Oncol 2013, 10:400-410.

3. Lehtinen M, French KM, Dillner J, Paavonen J, Garnett G: Sound implementation of human papillomavirus vaccination as a communityrandomized trial. In Future Medicine Ltd ISSN 1475-0708. Therapy; 2008:289-294.

4. Stanley M: Perspective: Vaccinate boys too. Nature 2012, 488:S10. 7413.

5. Petäjä T, Keränen H, Karppa T, Kawa A, Lantela S, Siitari-Mattila M, Levänen H, Tocklin T, Godeaux O, Lehtinen M, Dubin G: Immunogenicity and safety of human papillomavirus (HPV)-16/18 AS04-adjuvanted vaccines in healthy boys aged 10-18 years. J Adolesc Health 2009, 44:33-40.

6. Petäjä T, Pedersen C, Poder A, Strauss G, Catteau G, Thomas F, Lehtinen M, Descamps D: Long-term persistence of systemic and mucosal immune response to HPV-16/18 AS04-adjuvanted vaccine in preteen/adolescent girls and young women. Int J Cancer 2011, 129:2147-2157.

7. Dessy FJ, Giannini SL, Bougelet CA, Kemp TJ, David MP, Poncelet SM, Pinto LA, Wettendorff MA: Correlation between direct ELISA single epitope-based inhibition ELISA and pseudovirion-based neutralization assays for measuring anti-HPV16 and anti-HPV18 antibody response after vacination with the AS04-adjuvanted HPV-16/18 cervical cancer vaccine. Hum Vaccin 2008, 4:425-434.

8. Draper E, Bissett SL, Howell-Jones R, Edwards D, Munslow G, Soldan K, Beddows S: Neutralization of non-vaccine human papillomavirus pseudoviruses from the A7 and A9 species groups by bivalent HPV vaccine sera. Vaccine 2011, 29:8585-8590.

9. Roteli-Martins CM, Naud P, De Borba P, Teixeira JC, De Carvalho NS, Zahaf T, Sanchez N, Geeraerts B, Descamps D: Sustained immunogenicity and efficacy of the HPV-16/18 AS04-adjuvanted vaccine: up to 8.4 years of follow-up. Hum Vaccin Immunother 2012, 8:390-397.

10. Kemp TJ, Safaeian M, Hildesheim A, Pan Y, Penrose KJ, Porras C, Schiller JT, Lowy DR, Herrero R, Pinto LA: Kinetic and HPV infection effects on cross-type neutralizing antibody and avidity responses induced by Cervarix. Vaccine 2012, 31:165-170.

11. Scherpenisse M, Schepp RM, Mollers M, Meijer CJ, Berbers GA, van der Klis FR: Characteristics of HPV-specific antibody responses induced by Infection and Vaccination: cross-reactivity, neutralizing activity, avidity and IgG Subclasses. PLoS One 2013, 8:e74797.

12. Simen-Kapeu A, Kataja V, Yliskoski M, Syriänen K, Dillner J, Koskela P, Paavonen J, Lehtinen M: Smoking impairs human papillomavirus (HPV) type 16 and 18 capsids antibody response following natural HPV infection. Scand J Infect Dis 2008, 40:745-751.

13. Szarewski A, Jarvis JJ, Sasieni P, Anderson M, Edwards R, Steele SJ, Guillebaud J, Cuzick J: Effect of smoking cessation on cervical lesion size. Lancet 1996, 347:941-943.

14. Kapeu AS, Luostarinen T, Jellum E, Dillner J, Hakama M, Koskela P, Lenner $P$, Löve A, Mahlamaki E, Thoresen S, Tryggvadottir L, Wadell G, Youngman L, Lehtinen M: Is smoking an independent risk factor for invasive cervical 
cancer? a nested case-control study in Nordic biobanks. Am J Epidemiol 2009, 69:480-488.

15. Cruijff M, Thijs C, Govaert T, Aretz K, Dinant GJ, Knottnerus A: The effect of smoking on influenza, influenza vaccination efficacy and on the antibody response to influenza vaccination. Vaccine 1999, 17:426-432.

16. Lehtinen M, Paavonen J, Wheeler CM, Jaisamrarn U, Garland SM, Castellsagué X, Skinner SR, Apter D, Naud P, Salmerón J, Chow SN, Kitchener H, Teixeira JC, Hedrick J, Limson G, Szarewski A, Romanowski B, Aoki FY, Schwarz TF, Poppe WA, De Carvalho NS, Germar MJ, Peters K, Mindel A, De Sutter P, Bosch FX, David MP, Descamps D, Struyf F, Dubin G, for the HPV PATRICIA Study Group: Overall efficacy of HPV-16/18 AS04-adjuvanted vaccine against grade 3 or greater cervical intraepithelial neoplasia: 4-year end-of-study analysis of the randomised, double-blind PATRICIA trial. Lancet Oncol 2012, 13:89-99.

17. Paavonen J, Jenkins D, Bosch FX, Naud P, Salmerón J, Wheeler CM, Chow SN, Apter DL, Kitchener HC, Castellsague X, de Carvalho NS, Skinner SR, Harper DM, Hedrick JA, Jaisamrarn U, Limson GA, Dionne M, Quint W, Spiessens B, Peeters P, Struyf F, Wieting SL, Lehtinen MO, Dubin G, for the HPV PATRICIA study group: Efficacy of a prophylactic adjuvanted bivalent L1 virus-like particle vaccine against infection with human papillomavirus types 16 and 18 in young women: an interim analysis of a phase III double-blind randomised controlled trial. Lancet 2007, 369:2161-2170.

18. Dillner J, Kallings I, Brihmer C, Sikström B, Koskela P, Lehtinen M, Schiller JT, Sapp M, Mårdh PA: Seropositivity to human papillomavirus types 16,18 , or 33 capsids and to Chlamydia trachomatis are markers of sexual behaviro. J Infect Dis 1996, 173:1394-1398.

19. Simen-Kapeu A: Epidemiological study of tobacco use and human papillovirus, Implication for public health prevention. Academic dissertation. National Institute for Health and Welfare, Research 20, THL; 2009.

20. Namujju PB, Surcel HM, Kirnbauer R, Kaasila M, Banura C, Byaruhanga R, Muwanga M, Mbidde EK, Koskela P, Lehtinen M: Risk of being seropositive for multiple human papillomavirus types among Finnish and Ugandan women. Scand J Infect Dis 2010, 42:522-526.

21. Namujju PB, Hedman L, Hedman K, Banura C, Mbidde EK, Kizito D, Byaruhanga RN, Muwanga M, Kirnbauer R, Surcel HM, Lehtinen M: Low avidity of human papillomavirus (HPV) type 16 antibodies is associated with increased risk of low-risk but not high-risk HPV type prevalence. BMC Res Notes 2011, 4:170,

22. Hedman K, Lappalainen M, Seppälä I, Mäkelä O: Recent primari toxoplasma infection indicated by a low avidity of specific lgG. J Infect Dis 1989, 159:736-740.

23. Hedman L, Söderlund-Venermo M, Jartti T, Ruuskanen O, Hedman K: Dating of human bocavirus infection with protein-denaturing IgG-avidity assays - Secondary immune activations are ubiquitous in immunocompetent adults. J Clin Virol 2010, 48:44-48

24. Korhonen $\mathrm{MH}$, Brunstein J, Haario H, Katnikov A, Rescaldani R, Hedman K: A new method with general diagnostic utility for the calculation of immunoglobulin G avidity. Clin Diagn Lab Immunol 1999, 6:725-728.

25. Parish S, Collins R, Peto R, Youngman L, Barton J, Jayne K, Clarke R, Appleby P, Lyon V, Cederholm-Williams S, Marshall J, Sleight P for the International Studies of Infarct Survival (ISIS) Collaborators: Cigarette smoking, tar yields, and non-fatal myocardial infarction: 14000 cases \& 32000 controls in the United Kingdom. BMJ 1995, 311:471-477.

26. Pérez-Stable EJ, Benowitz NL, Marín G: Is serum cotinine a better measure of cigarette smoking than self-report? Prev Med 1995, 24:171-179.

27. Stanley M, Lowy DR, Frazer I: Chapter 12: Prophylactic HPV vaccines: underlying mechanisms. Vaccine 2006, 24S3:106-113.

28. Giannini SL, Hanon E, Moris P, Van Mechelen M, Morel S, Dessy F, Fourneau MA, Colau B, Suzich J, Losonksy G, Martin MT, Dubin G, Wettendorff MA: Enhanced humoral and memory B cellular immunity using HPV16/18 L1 VLP vaccine formulated with the MPL/aluminium salt combination (AS04) compared to aluminium salt only. Vaccine 2006, 24:5937-5949.

29. Einstein MH, Baron M, Levin MJ, Chatterjee A, Fox B, Scholar S, Rosen J, Chakhtoura N, Meric D, Dessy FJ, Datta SK, Descamps D, Dubin G, and on behalf of the HPV-010 Study group: Comparative immunogenicity and safety of human papillomavirus (HPV)-16/18 vaccine and HPV-6/11/16/18 vaccine: follow-up from months 12-24 in a Phase III randomized study of healthy women aged 18-45 years. Hum Vaccin 2011, 7:1343-1358.

30. af Geijersstam V, Kibur M, Wang Z, Koskela P, Pukkala E, Schiller J, Lehtinen M, Dillner J: Stability over time of serum antibody levels to human papillomavirus type 16. J Infect Dis 1998, 176:1710-1714.
31. Dauner JG, Pan Y, Hildesheim A, Kemp TJ, Porras C, Pinto LA: Development and application of a GuHCl-modified ELISA to measure the avidity of anti-HPV L1 VLP antibodies in vaccinated individuals. Mol Cell Probes 2012, 26:73-80.

32. Barton SE, Maddox PH, Jenkins D, Edwards R, Cuzick J, Singer A: Effect of cigarette smoking on cervical epithelial immunity: a mechanism for neoplastic change? Lancet 1988, 2:652-654.

33. Szarewski A, Maddox P, Royston P, Jarvis M, Anderson M, Guillebaud J, Cuzick J: The effect of stopping smoking on cervical Langerhans' cells and lymphocytes. BJOG 2001, 108:295-303.

34. Syrjänen K, Shabalova I, Petrovichev N, Kozachenko V, Zakharova T, Pajanidi J, Podistov J, Chemeris G, Sozaeva L, Lipova E, Tsidaeva I, Ivanchenko O, Pshepurko A, Zakharenko S, Nerovjna R, Kljukina L, Erokhina O, Branovskaja M, Nikitina M, Grunberga V, Grunberg A, Juschenko A, Santopietro R, Cintorino M, Tosi P, Syrjänen S: Smoking is an independent risk factor for oncogenic human papillomavirus (HPV) infections but not for high-grade CIN. Eur J Epidemiol 2007, 22:723-735.

35. Giuliano AR, Sedjo RL, Roe DJ, Harri R, Baldwi S, Papenfuss MR, Abrahamsen M, Inserra P: Clearance of oncogenic human papillomavirus (HPV) infection: effect of smoking (United States). Cancer Causes Control 2002, 13:839-846.

36. Murray RP, Connett JE, Lauger GG, The Lung Health Study Research Group: Voelker HTError in smoking measures: effects of intervention on relations of cotinine and carbon monoxide to self-reported smoking. Am J Public Health 1993, 83:1251-1257.

37. Murray RP, Connett JE, Istvan JA, Nides MA, Rempel-Rossum S: Relations of cotinine and carbon monoxide to self-reported smoking in a cohort of smokers and ex-smokers followed over 5 years. Nicotine Tob Res 2002, 4:287-294.

38. Boffetta P, Clark S, Shen M, Gislefoss R, Peto R, Andersen A: Serum cotinine level as predictor of lung cancer risk. Cancer Epidemiol Biomarkers Prev 2006, 15:1184-1188.

39. Olsson SE, Villa LL, Costa RL, Petta CA, Andrade RP, Malm C, Iversen OE, Høye J, Steinwall M, Riis-Johannessen G, Andersson-Ellstrom A, Elfgren K, von Krogh G, Lehtinen M, Paavonen J, Tamms GM, Giacoletti K, Lupinacci L, Esser MT, Vuocolo SC, Saah AJ, Barr E: Induction of immune memory following administration of a prophylactic quadrivalent HPV types 6/11/16/ 18 L1 virus-like particle (VLP) vaccine. Vaccine 2007, 25:4931-4939.

doi:10.1186/1756-0500-7-445

Cite this article as: Namujju et al.: Impact of smoking on the quantity and quality of antibodies induced by human papillomavirus type 16 and 18 AS04-adjuvanted virus-like-particle vaccine - a pilot study. BMC Research Notes 2014 7:445

\section{Submit your next manuscript to BioMed Central and take full advantage of:}

- Convenient online submission

- Thorough peer review

- No space constraints or color figure charges

- Immediate publication on acceptance

- Inclusion in PubMed, CAS, Scopus and Google Scholar

- Research which is freely available for redistribution 